\title{
PHOTOMETRIC DATA FROM SOME PHOTOGRAPHS OF MARS OBTAINED WITH THE AUTOMATIC INTERPLANETARY STATION 'MARS 3'
}

\author{
V. V. BOTVINOVA, O. I. BUGAENKO, I. K. KOVAL, M. K. NARAJEVA, \\ and
}

A. S. SELIVANOV

Main Astronomical Observatory, Kiev, U.S.S.R.

\begin{abstract}
The results of detailed photometric treatment of Mars photographs obtained with the Automatic Interplanetary Station 'Mars 3' in three wavelengths are given. Photometric maps of the Martian surface have been constructed; a thin layer observed near the limb has been investigated.
\end{abstract}

As is well known (Pravda, 1971), the scientific equipment on the Soviet automatic interplanetary stations 'Mars 2' and 'Mars 3' included phototelevision devices for obtaining both a limited number of whole-disk planetary photographs in the visible region of the spectrum, and photographs of separate regions of the Martian surface. According to the program the photographic investigations were mainly executed by 'Mars 3', whose orbit allowed surveys of the planet from both small and relatively great distances (distance in periares $1500 \mathrm{~km}$, distance in apoares $200000 \mathrm{~km}$ ). Photography of the Martian surface has been also made at phases not observed from the Earth. Each of the stations had two phototelevision devices (FTU) equipped with objectives having focal distances $F=52 \mathrm{~mm}$ (FTU I) and $F=350 \mathrm{~mm}$ (FTU II). In front of the wideangle objective FTU I was a wheel with three colour filters (Figure 1). The picture taking has been carried out cyclically at the rate of 140 or $35 \mathrm{~s}$ and with 12 frames in a cycle.

In the television instruments the pictures were recorded on film (width $25.4 \mathrm{~mm}$, frame size $24 \times 24 \mathrm{~mm}$ ), with subsequent chemical processing. The image obtained was read out by an optical-mechanical television device. The number of lines in a frame can be set equal to 64,250 , or 1000 .

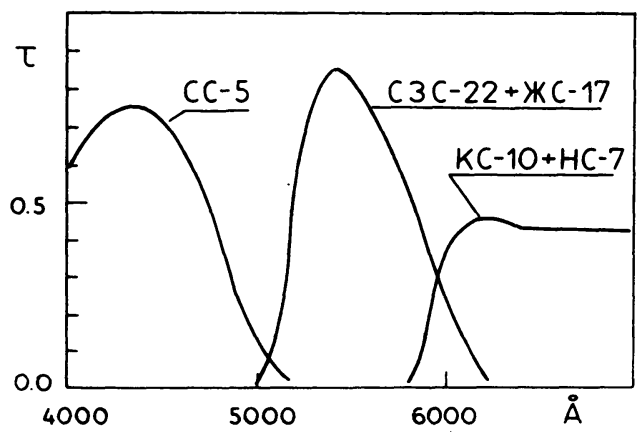

Fig. 1. Graphs of filter transmissions used in FTU II of 'Mars 3'. 
A series of Mars pictures taken by 'Mars 3' on February 28, 1972, at a distance of $18000 \mathrm{~km}$, has been selected for detailed photometric study. These photographs were of interest because of the phase angle $\left(\alpha \approx 103^{\circ}\right)$, at which the Martian surface has not been observed before. Besides, the photographs showed that one of the Martian cusps had a weakly visible thin haze next to the limb, that rapidly decreased in brightness off the limb. The phenomenon was similar to those observed with 'Mariner 6, 7' (Leovy et al., 1971) and 'Mariner 9' (Masursky et al., 1972).

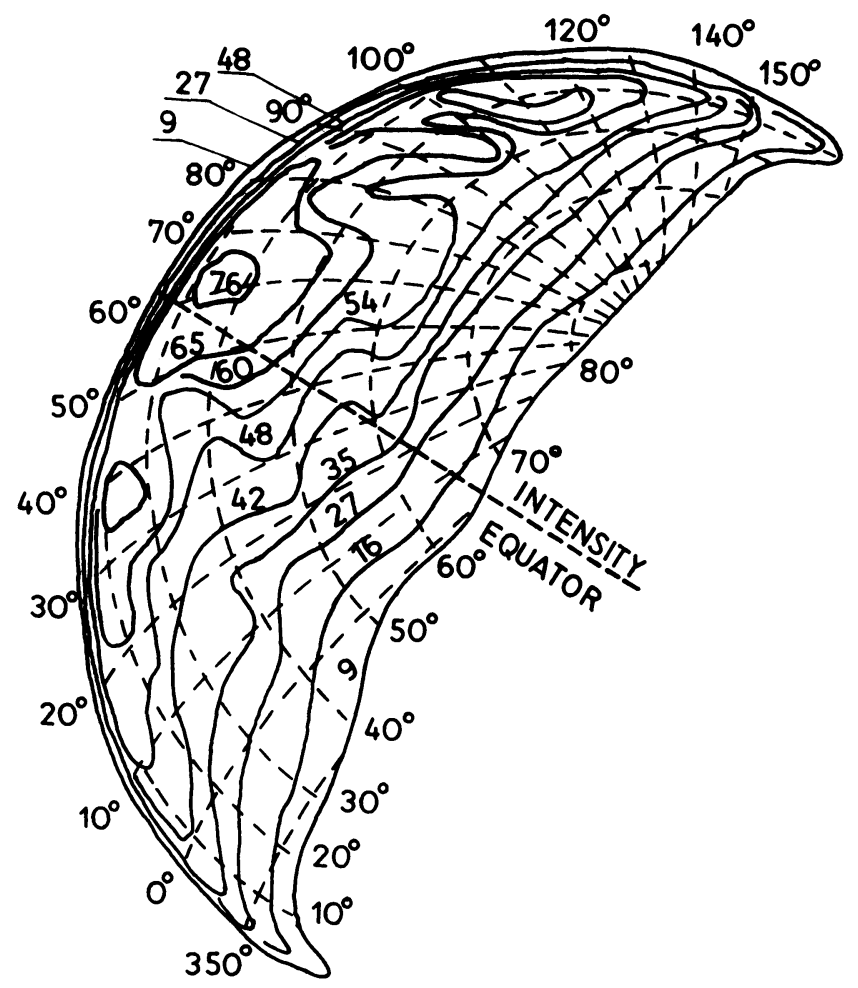

Fig. 2. Photometric map of Mars obtained on February 28, 1972 (red filter).

On the ground of some considerations the photometric analysis of the Martian surface has been carried out not on the pictures themselves but using the phototelevision lines recorded on the band of the loop oscillograph.

From photometric treatment of the selected pictures, we got the photometric maps of Mars for three spectral regions, presented (Botvinova et al., 1974) as isophotes (relative intensities in Figure 2). From these maps, the authors estimated the contrast between Mare Acidalium and a continental region placed symmetrically with respect to the intensity equator. In red light the contrast appeared to be 0.25 (lower limit) which agrees with ground-based measurements (Barabashov and Koval, 1959; de Vaucouleurs, 1967). In blue light the contrast reaches 0.20 and has the opposite sign because of the cloudy formation extending over Mare Acidalium. 


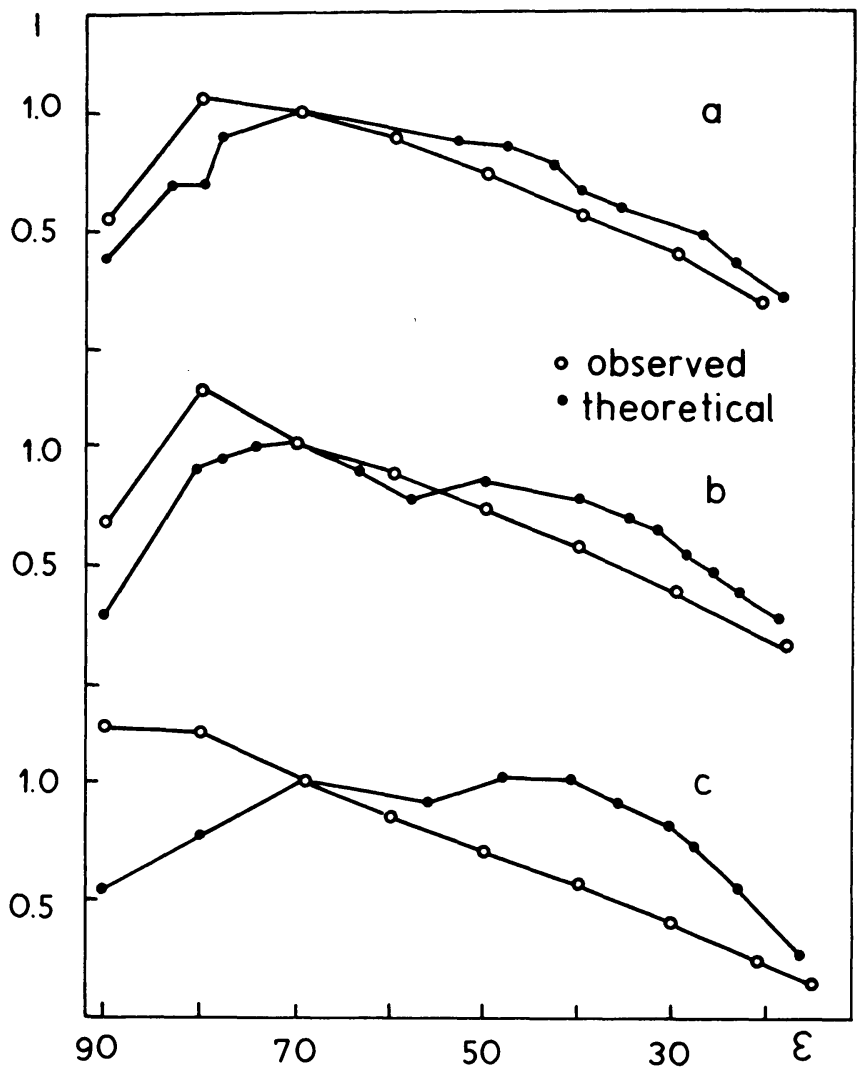

Fig. 3. Brightness distribution along the Mars intensity equator obtained on February 28, 1972. $\mathrm{a}$ - red filter, $\mathrm{b}$ - green filter, $\mathrm{c}$ - blue filter.

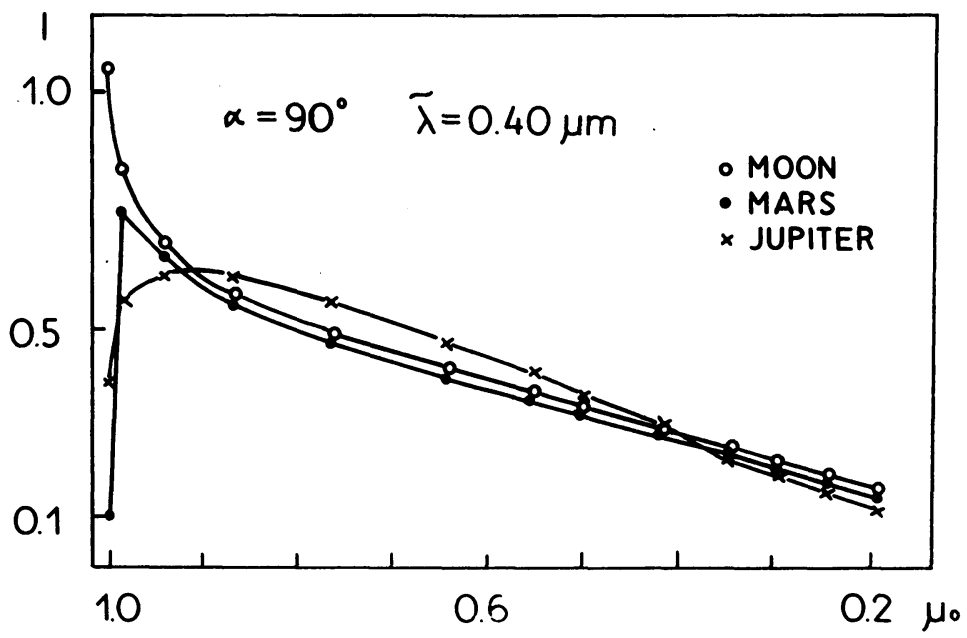

Fig. 4. Calculated distribution of brightness along the intensity equator of Moon, Mars and Jupiter $\left(\mu_{0}=\cos \varepsilon\right)$. 
For the same pictures the graphs of brightness variation from limb to terminator were plotted. These were compared with theoretical intensity curves calculated by Morozhenko and Yanovitsky (1971) on the assumption that the Hapke model is true for the Martian surface (Figure 3). The comparison showed satisfactory agreement between the theoretical and observed curves in red and blue light (within the limits of measuring errors and the approximate character of the theory). In blue light the calculated curve differs from the observed one mainly due to the cloudy formation in the centre of the picture. The brightness distribution along the Martian intensity

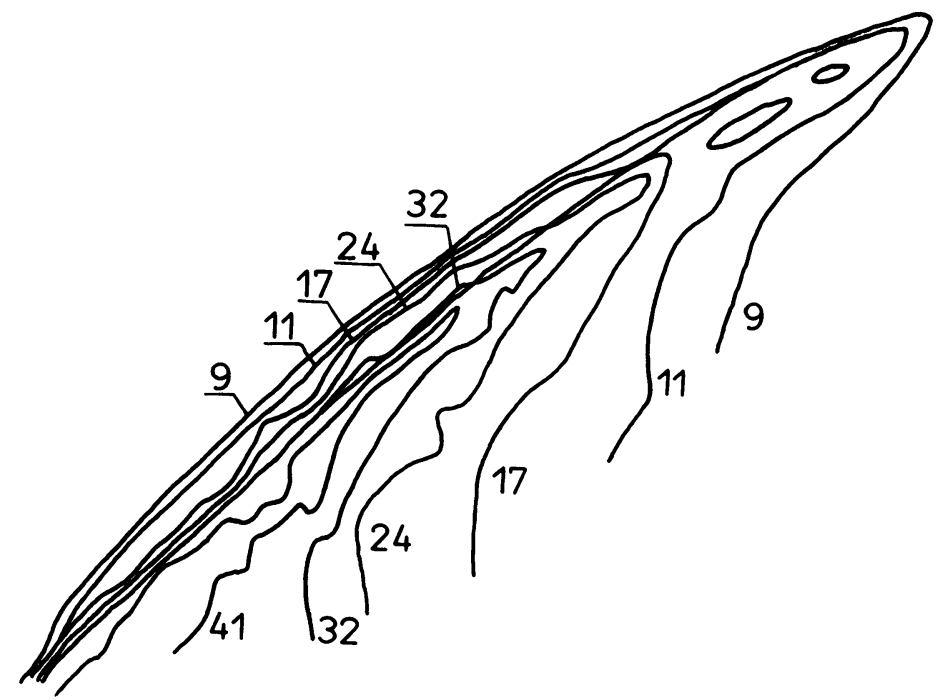

Fig. 5. Photometric map of a cusp with cloud formation in the limb zone (red filter).

equator when $\alpha \approx 103^{\circ}$ is not very informative. However, its comparison with a theoretical model which is in a good agreement with observations obtained with 'Mariner 6, 7' (Hord, 1972) gives an additional criterion for estimating the accuracy of the photographic experiment obtained with 'Mars 3' (Figure 4).

Study of the cloudy formation near a cusp showed that its upper limit is at $30-40$ $\mathrm{km}$ and its extension along the limb reaches 20 areographic degrees. From detailed photometry of a cusp the isophotes of the cloudy formation and the surface border Figure 5 have been constructed. The authors have estimated the marked decrease of the cloud brightness relative to the planetary limb when passing from red to blue.

To study the distribution of cloud intensity with height, six photometric sections were made in each of the three filters along the radius of the image. The measurements were reduced to absolute units by tying a photometric section made along the planetary intensity equator to the theoretical ones, calculated by Morozhenko and Yanovitsky for the usual case of high transparency of the Martian atmosphere. Analysing the height profiles of the Martian atmosphere, one can conclude that the scattering particles forming the cloud at different heights have the same size spectrum, 
if this cloud is optically thin and its particles are purely scattering (Figure 6). The intensity relations of a cloud in blue and green light to that in the red (Figure 7) showed that the cloud formation, throughout its height, had evidently selective reflection, i.e., its brightness in the red is three or four times more than in the blue. This means that the cloud under investigation consists of large particles throughout its height (according to the above model).

Qualitative characteristics of particles in the cloud formations under investigation (mean radius, the number in vertical cohumn of atmosphere) can be estimated only from an investigation of the spectral dependence of their optical thickness. But this is the subject of another study.

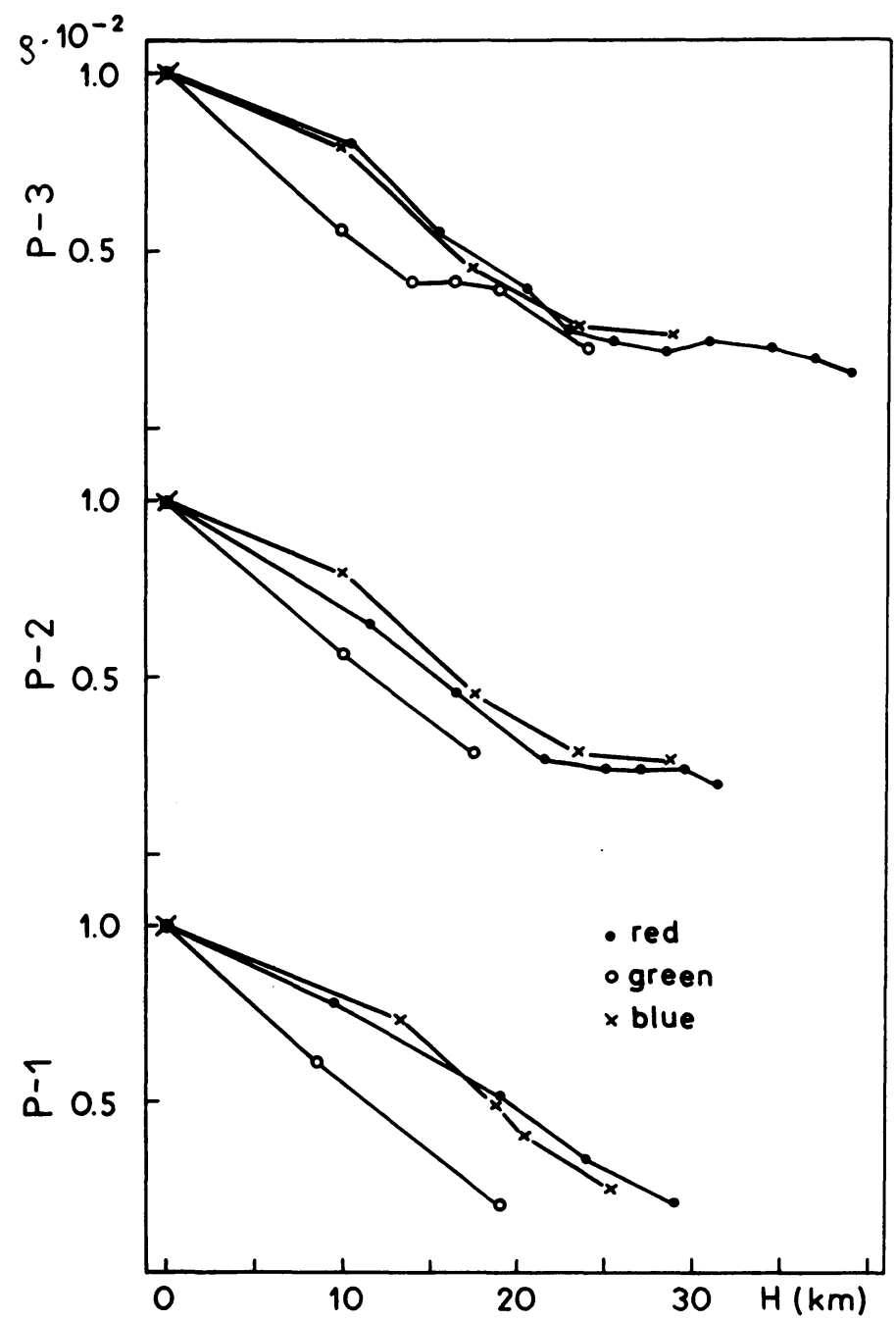

Fig. 6. Height-brightness profiles of different regions of cloud formations in a cusp limb zone (graphs photometrically connected near the base of the cloud). 


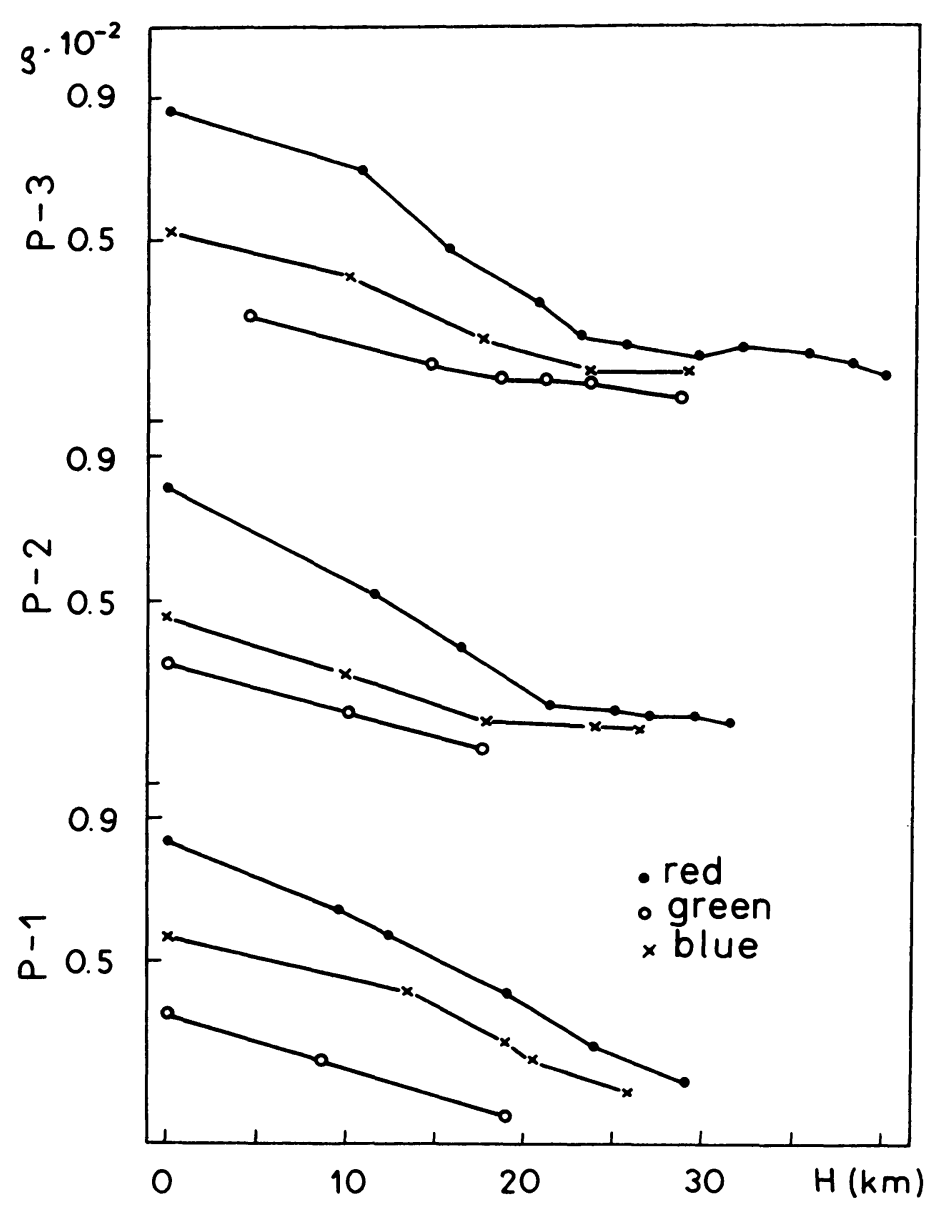

Fig. 7. Height-brighntess profiles of different regions of the cloud formations in the cusp limb zone (in absolute units).

\section{References}

Barabashov, N. P. and Koval, I. K.: 1959, Photographic Photometry of Mars with Colour Filters in 1956, Izd. Kharkiv. Univ. (in Russian).

Botvinova, V. V., Bugaenko, O. I., Koval, I. K., Narajeva, M. K., and Selivanov, A. S.: 1974, Photometric Data from Photographs Obtained with 'Mars 3' on February 28, 1972 (in Russian), in press

De Vaucouleurs, G.: 1967, Icarus 7, 310.

Hord, C. W. 1972, Icarus 16, 253.

Leovy, C. B., Smith, B. A., Young, A. T., and Leighton, R. B.: 1971, J. Geophys. Res. 76, 297.

Masursky, H., Batson, R. M., McCauley, J. F., Soderblom, L. A., Wildey, R. L., Carr, M. H., Milton, D. J., Wilhelms, D. E., Smith, B. A., Kirby, T. B., Robinson, J. C., Zeovy, C. B., Briggs, G. A., Duxbury, T. C., Acton, C. H., Jr., Murray, B. C., Cutts, J. A., Sharp, R. P., Smith, S., Leighton, R. B., Sagan, C., Veverka, J., Norald, M., Zederberg, J., Zevinthal, E., Pollack, J. B., Moore, J. T., Jr., Hartmann, W. K., Shipley, E. N., de Vaucouleurs, G., and Davies, M. E.: 1972, Science 175, 294.

Morozhenko, A. V. and Yanovitsky, E. G.: 1971, Astron. Zh. 48, 798.

Pravda: 1971, No. 353, 2 (in Russian) 